\title{
Problemas en la transposición didáctica para un sistema clásico y uno cuántico: el caso de la partícula en una caja
}

Didactic transposition problems for a classical system and a quantum one: the case of the particle in a box

\author{
C. H. Wörner ${ }^{* 1}$ \\ ${ }^{1}$ Pontificia Universidad Católica de Valparaíso, 2340000, Valparaíso, Chile.
}

Recibida en 10 de Enero, 2022. Aceptado en 27 de Enero, 2022.

\begin{abstract}
Este trabajo examina el modelo de una partícula clásica/cuántica confinada en una caja unidimensional. En términos clásicos se describen sus propiedades cinemáticas; posición, velocidad y aceleración. En un escenario cuántico, estas características están ausentes. Pretendemos discutir la posible transposición didáctica necesaria para introducir su tratamiento cuántico. Los resultados se presentan como una sugerencia con fines didácticos; en particular para el caso de alguna dificultades en la transposición didáctica.

Palabras-clave: Enseñanza de la física, mecánica cuántica, transposición didáctica.
\end{abstract}

This work examines the model of a classical/quantum particle confined in a one-dimensional box. In classical terms its kinematic properties: position, velocity and acceleration are described. In a quantum setting, these characteristics are absent. We intend to discuss the possible didactic transposition necessary to introduce its quantum treatment. The results are presented as a suggestion for educational purposes; in particular for the case of some difficulties on the didactic transposition.

Keywords: Physics teaching, quantum mechanics, didactic transposition.

\section{Introducción}

En una serie de artículos, en su año quizás más creativo, 1926, Erwin Schrödinger [1] describe su hallazgo de lo que hoy se llama "ecuación de Schrödinger", el fundamento básico de la mecánica cuántica. En cierto sentido, el trabajo de Schrödinger contiene una introducción a las estrategias que siguió para encontrar esta ecuación, que proporcionaría una plausibilidad para la exactitud de este cálculo antes mencionado. De esta manera, el exponía una plataforma sobre la cual sustentar su hallazgo.

Así, esta exposición de Schrödinger puede proporcionar una base sobre la cual construir la ingeniería didáctica apropiada para pasar desde un sistema clásico a uno cuántico. También recientemente, Karam 2 describe los conflictos epistemológicos que enfrentó Schrödinger al considerar la función de onda como una magnitud compleja y algunas de las consecuencias para su enseñanza.

No hay nada nuevo bajo el sol. Los filósofos griegos utilizan algunas estrategias didácticas para enseñar sus conocimientos y, en el Renacimiento, Galileo en una de sus obras esenciales [3], nos enseñó la cinemática a través de un supuesto diálogo con sus discípulos (utilizando una especie de teoría del constructivismo).

Recientemente, Chevallard 4,5] propuso la distinción entre conocimiento académico (o sabio) y conocimiento

\footnotetext{
${ }^{*}$ Correo electrónico: carlos.worner@pucv.cl
}

enseñado. Postuló que existe una diferencia entre estas dos formas de describir la realidad y que un docente debe ser consciente de esta transición del conocimiento científico al conocimiento enseñado (por ejemplo, como aparece en los libros de texto).

El primer campo de aplicación de estas ideas fue la enseñanza de las matemáticas. Un buen relato de esta forma de pensamiento aparece en Klisinska 66. Básicamente, esta es la esencia de la "teoría de la transposición didáctica".

En esta transposición hay que tener cuidado para mantener la esencia del conocimiento transferido, especialmente cuando el dominio de la enseñanza escapa a las intuiciones del alumno. Recientemente se ha publicado un nuevo avance sobre la importancia de estas intuiciones en el proceso enseñanza/aprendizaje [7].

No hace falta decir que este es el caso de la didáctica de la mecánica cuántica. En este trabajo manejaremos la transición de un tratamiento mecánico clásico a uno cuántico sobre el problema simple: la partícula en una caja. El tratamiento cuántico estándar de este tema se puede encontrar en manuales de introducción a la mecánica cuántica, por ejemplo, el conocido texto escrito por Serway et al. 8 .

El interés de este problema radica en el hecho que es el problema cuántico más simple de resolver por métodos analíticos. Por tanto, se utiliza de forma didáctica para enseñar a calcular los valores propios de la energía y las funciones propias correspondientes. Lo anterior, teniendo debidamente en cuenta las condiciones de contorno 
del problema. Artículos recientes 9 12, muestran el interés contemporáneo en la enseñanza de la Mecánica Cuántica a un nivel elemental, en especial el trabajo de Winkler et al. que trata de encontrar cuáles son los tópicos que deben incluirse en un estudio elemental de la física cuántica 13 .

Una de las maneras de hacer la transposición didáctica es recurrir a la historia del descubrimiento en cuestión. De esta manera se presenta el problema epistemológico que intenta resolver el científico. En este sentido el análisis de Schrödinger, descrito en la Ref. 1, es poco apropiado por su complejidad para el caso que nos preocupa, un curso de enseñanza introductoria de la física.

En este trabajo describiremos primero el tratamiento clásico y luego el cuántico para la partícula en una caja unidimensional. Así, compararemos diferencias y semejanzas.

\section{El Caso Clásico}

Inicialmente describiremos - en su forma newtoniana unidimensional - el movimiento sin roce de una partícula clásica de masa $m$ con velocidad $v$ en una región limitada del eje $X$, con longitud $L$. En los límites de este movimiento restringido, hay paredes impenetrables (ver Figure 1).

En realidad, la descripción del movimiento se obtiene mediante simple inspección. El enfoque mecánico habitual que utiliza la ley de Newton es engorroso. En cambio, es más fácil comenzar con una descripción simple de la velocidad: así este tratamiento es mucho más intuitivo. Definamos arbitrariamente $t=0$ como el instante en que la partícula acaba de comenzar a moverse en la dirección $+X$ desde $x=0$. Entonces, la velocidad se expresa por,

$$
v=\left\{\begin{array}{l}
+v, \quad 0<t<\frac{L}{v} \\
-v, \quad \frac{L}{v}<t<\frac{2 L}{v}, \\
v\left(t+\frac{2 L}{v}\right)=v(t)
\end{array}\right.
$$

con las condiciones de contorno: $x(0)=0 ; \dot{x}(0)=v$ (constante).

Como consecuencia, el gráfico de velocidad tiene la forma de una "onda cuadrada". Tengamos en cuenta

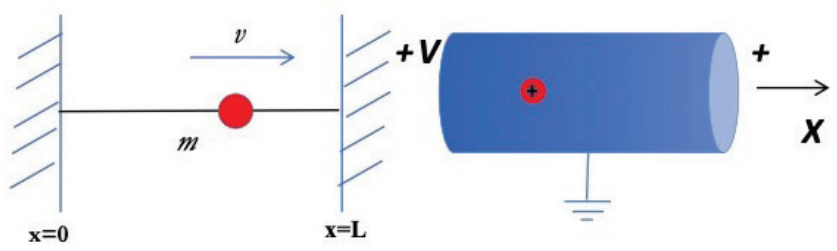

Figura 1: Una partícula de masa $m$ está atrapada en una pared de largo $L$. A la derecha se observa una carga puntual oscilando entre las paredes de un conductor cilíndrico con extremos equipotenciales. que hay discontinuidades finitas en los puntos $t=$ $\ldots,-L / v, 0, L / v, \ldots$ Este hecho es consistente con el supuesto que la interacción de la pelota con las paredes es perfectamente elástica, sin pérdida de energía. Claramente, no es un hecho efectivamente realista, pero es el costo que se debe pagar para mantener la simplicidad del modelo.

Continuando con la descripción, el diagrama $(x-t)$ muestra una forma de "diente de sierra", con derivadas discontinuas en los puntos donde impacta la partícula, es decir, puntos que corresponden a saltos de velocidad.

La dependencia del tiempo de la aceleración y en consecuencia, de la fuerza, debe cumplir con las leyes de Newton. Así, en el punto de contacto con la pared, la aceleración no es una función bien definida. En este momento, la debilidad del modelo es evidente y por lo tanto una oportunidad para discutir con los estudiantes sobre las limitaciones de éste o cualquier modelo.

El caso que nos ocupa es un modelo de situación física. Por lo tanto, al usarlo para construir otro modelo (es decir, el pozo cuántico), parece un "caso de dos modelos". Quizás, aceptamos implícitamente esta plausibilidad debido al hecho de que en la enseñanza de la física elemental usualmente usamos esta situación clásica para introducir el modelo de gas ideal. De esta manera, seguimos hacia la ecuación de estado y la expresión cinética de la energía (interna). Allí, de nuevo aparece la vía de la transposición didáctica.

\section{El Caso Cuántico}

Posiblemente la ubicuidad de este tema en la práctica actual de la enseñanza de la física se deba a la formulación sencilla de la ecuación de Schrödinger para este caso. Además, la solución de la ecuación diferencial es un simple ejercicio analógico con raíces en la mecánica ondulatoria newtoniana. Como señaló Feynman [14]: ...las mismas ecuaciones tienen las mismas soluciones.

Recordemos la introducción: estamos tratando con un problema unidimensional formulado para una función potencial:

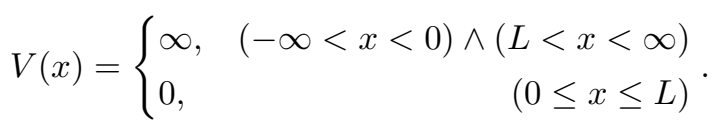

De este modo, la función de onda $(\psi)$, queda determinada por $\frac{d^{2} \psi}{d x^{2}}+\frac{\hbar^{2} E}{2 m} \psi=0$; para $(0 \leq x \leq L)$, que es la bien estudiada ecuación del oscilador armónico unidimensional. También, $\psi \equiv 0$ en el resto del espacio.

Entonces su conocida solución [8] es,

$$
\psi(x)=\left\{\begin{array}{l}
0(-\infty<x<0) \wedge(L<x<\infty) \\
\mathrm{A} \sin \left(\sqrt{\frac{2 m E_{n}}{\hbar^{2}}} x\right) \quad 0 \leq x \leq L
\end{array},\right.
$$

con los autovalores

$$
E_{n}=\frac{\pi^{2} \hbar^{2}}{2 m L^{2}} n^{2} ; \quad n \in \mathbb{N}^{+} \text {, es el número cuántico. }
$$


De esta manera, la cuantización de los niveles de energía, aparece nítidamente de la condición de tener soluciones finitas $\forall x$.

\section{La Ruta de Transposición para Este Tema}

Para analizar mejor esta cuestión en el contexto de la transposición didáctica, es aclarador señalar (en un orden arbitrario) dos formas de hacer la transición del conocimiento docto al didáctico. Estas formas se pueden rastrear por el desarrollo histórico de la mecánica cuántica y son la forma habitual de enseñar este tema.

Esto no significa hacer una taxonomía del proceso didáctico. Más bien, su uso es para simplificar el análisis. En la forma habitual de tratar este tránsito didáctico, hay una mezcla de estos caminos al modo que el instructor fija (muchas veces de manera implícita), según su percepción sobre la audiencia, profundidad, resultados esperados, etc.

Cabe señalar que la correspondiente reflexión epistemológica debe ser previamente formulada y reforzada. La ciencia física es una disciplina experimental cuyo índice de verdad (al menos provisional) es el resultado de experimentos.

i) El camino pre-cuántico (Rutherford, Bohr y De Broglie)

Lo que aquí llamamos el camino pre-cuántico es básicamente la formulación de Bohr que él aplica al tratamiento del átomo de hidrógeno. En este modelo, Bohr recoge la hipótesis planetaria de Rutherford y la propuesta de Planck y Einstein sobre la energía del cuanto de luz (fotón). Rompiendo con la Física clásica, postula la existencia de órbitas privilegiadas (aquellas en las que el momento angular es un múltiplo entero de la constante de Planck). En ellas, contrariamente al electromagnetismo clásico, el sistema no irradia. Para satisfacer los resultados espectroscópicos, también postula que el sistema irradia a través de "saltos cuánticos" entre órbitas de diferente energía. De esta manera, se puede encontrar un valor de la constante de Rydberg, dentro de los errores experimentales. Los detalles se pueden encontrar en cualquier libro de texto 8, 13. Claramente, esto no explica las hipótesis de Bohr y así aparece la ansatz de De Broglie, que postula que la partículas materiales presentan una longitud de onda $\lambda=h / p$, donde $h$ es la constante de Planck y $p$ es el momentum de la partícula.

Extendiendo este resultado, se puede hacer una analogía con el problema que hoy nos preocupa. Se puede establecer una similitud entre este problema y los modos normales de oscilación de una cuerda fija por ambos extremos. Los modos normales de este último problema, se determinan por las relaciones $L=n \frac{\lambda}{2}, n \in \mathbb{N}^{+}$; de este modo, poniendo $L=2 \pi r$ y usando la hipótesis de De Broglie, se obtiene el radio de las órbitas privilegiadas que aparecen ad-hoc en el tratamiento de Bohr.
Este enfoque puede usarse para hacer una transposición didáctica que recoge las siguientes noveles ideas:

1. La física clásica no permite un tratamiento coherente con los resultados experimentales. Quedan por consiguiente, dos soluciones: seguir buscando algún tratamiento que clásicamente explique los resultados espectroscópicos o bien construir una nueva teoría que reconcilie teoría con experimento. (Esta situación histórica/epistemológica, ha aparecido antes en el desarrollo de las ciencias experimentales, por ejemplo, el desechar la teoría del calórico, o la intuición de Einstein al destruir el concepto de éter luminífero).

2. En un modo didáctico, permite aprehender varios conceptos cuánticos: la cuantización de la energía o la inexistencia de movimiento (la partícula no puede estar "quieta" y esto puede enlazarse con el principio de incertidumbre de Heisenberg).

ii) El tratamiento de Schrödinger

El tratamiento anterior, claramente influenció en la intuición de Schrödinger, aunque el imaginó otro modo de aproximación. Directamente, el desarrollo de la ciencia nos lleva a concluir que - al no encontrar un camino clásico o semi-clásico - se desarrolla un nuevo modo que llamamos "cuántico" cuyo fundamento podemos encontrar en la ecuación de Schrödinger. La propuesta de Schrödinger es insólita e inesperada y suele usarse para ejemplificar las ideas epistemológicas de Kuhn [15]. En el trabajo de Schrödinger ya citado en la introducción [1], la idea seminal consiste en la aceptación de la hipótesis de De Broglie. Para decirlo de manera algo ramplona, el argumento usado sería: si hay una onda, debe haber una ecuación de onda. Schrödinger explica que usando la analogía geométrica para la óptica, es posible hacer un tratamiento ondulatorio y así llegar a la óptica ondulatoria, usando una formulación establecida por Hamilton. Una versión con mayor rigor y detalle ha aparecido recientemente 16 .

Henos aquí frente a un problema didáctico. Su intervención por el instructor crea un camino - que introducen los textos- de la versión de Bohr. Sin embargo, su aprendizaje significativo [17 puede representar la introducción de conceptos "erróneos". Por ejemplo: la asunción de que las partículas microscópicas (electrón, protón, etc.) son partículas esféricas, la existencia de trayectorias y en fin, una percepción clásica del mundo atómico.

En resumen, pareciera que la adaptación didáctica de este ejercicio es complicada.

Aparentemente, hay tres caminos para tratar esta cuestión.

i. Seguir el camino didáctico clásico: Rutherford, Bohr, De Broglie y analogías mecánicoondulatorias.

\footnotetext{
1 En estricto rigor, la ecuación de Schrödinger no puede ser "demostrada" a partir de primeros principios.
} 
ii. Proponer una crisis epistemológica y seguir un camino cuasi axiomático, postulando la ecuación de Schrödinger.

iii. Hacer una aproximación descriptiva y seguir con este nivel elemental, rescatando los principales resultados del proceso cuántico.

\section{Comentarios}

El tema en comento es un tema didáctico que también aparece en la mecánica clásica, donde la intuiciones ensombrecen la verdad científica (y la didáctica). Así aparecen los fantasmas de los preconceptos: distorsiones construidas en nuestro andar cognitivo por la vida infantil/juvenil. Hay una numerosa literatura que ha revisado el tema de los preconceptos en la enseñanza elemental y universitaria introductoria (ver por ejemplo, los trabajos de McDermott [18 y Vienott [19]). Al revés de esta transición cuántica-clásica, en este trabajo se supone que la componente clásica de la mecánica está aprehendida. En verdad, esta mezcla de preconceptos clásicos y el quiebre de los "verdaderos" paradigmas clásicos con la irrupción de un nuevo paradigma cuántico es una situación poco explorada en la literatura didáctica.

Muy brevemente, hay dos cuestiones que hacen que esta transposición sea compleja y complican la enseñanza de este tema: i) La naturaleza de la misma pregunta cuántica (ya dijo Feynmann 9 , ".. . creo con seguridad que nadie entiende la mecánica cuántica", es decir, ella carece de elementos intuitivos y por tanto elementos para una aproximación constructivista a la enseñanza/aprendizaje y ii) el nivel de desarrollo de los estudiantes a quienes están dirigido la enseñanza con una comprensión significativa, como lo señala Piaget [20].

¿Cómo enseñar contenidos que no tengan analogías clásicas? ¿Cómo enseñar materias sin analogías clásicas? Los resultados (de cada modalidad didáctica) deben concluir en la expresión que concuerde con los resultados experimentales. Este es el resultado final, universal y falseable. Es necesario tener concordancia dentro de las dos vías discutidas: la argumentación académica y didáctica.

También hay que tomar en cuenta que la aparición de ideas en los curricula ocurre con la enseñanza escolarizada de la química [21. En los textos de esta disciplina aparecen los conceptos de estados estacionarios, orbitales, saltos cuánticos con emisión de una fotón. Generalmente, la contribución de Schrödinger aparece de manera no-formal.

En opinión de quién esto escribe, la única "semejanza" entre el tratamiento clásico de este problema es la expresión de la energía potencial (ver Ecuación 2) y este lazo es muy feble para usarlo como un fundamento para la transposición didáctica.

Finalmente, quiero mencionar las introducciones a la mecánica cuántica de Wichmann 22 y Feynman mismo [14. El primero, construyendo un camino diferente a lo habitual para la construcción de la Ecuación de Schrödinger y el segundo elaborando una senda didáctica que podríamos llamar fenomenológica de aproximación a la mecánica cuántica.

Muy recientemente, Krijtenburg-Lewerissa et al. [23] han estudiado la influencia de la aprehensión del concepto de energía potencial clásica en el aprendizaje de la física cuántica. Su tesis es que una buena asimilación de la idea clásica favorece el aprendizaje de la física cuántica. Con su énfasis en la energía potencial (Ecuación 2) se destaca, que tanto la formulación clásica como la cuántica usan la misma expresión para la energía potencial. Así podría hacerse una aproximación didáctica usando clásicamente, $E=\frac{1}{2} m v^{2}$, y compararlo con la ecuación cuántica (Ecuación 4).

\section{Agradecimientos}

Quiero agradecer a mis alumnos que repetidamente me preguntaban: ¿Cómo se le ocurrió a Schrödinger esta ecuación? Así nacieron algunas de las reflexiones que describo en esta nota.

\section{Referencias}

[1] E. Schrödinger, Physical review 28, 1049 (1926).

[2] R. Karam, Am. J. Phys. 88, 433 (2020).

[3] G. Galileo, Two New Sciences (Dover, New York, 1954).

[4] Y. Chevallard, en: Proceedings of the international symposium on selected domains of research and development in mathematics education (Comenius University, Bratislava, 1989), p. 51.

[5] Y. Chevallard y M. Bosch, en: Encyclopedia of mathematics education, (Springer, Dordrecht, 2020), p. 214.

[6] A. Klisinska, The fundamental theorem of calculus: a case study into the didactic transposition of proof. Tesis de Doctorado, Luleå tekniska universitet, Luleå (2009).

[7] M. Vicovaro, European Review of Applied Psychology 71, 100610 (2021).

[8] R.A. Serway, C.J. Moses y C.A. Moyer, C A Modern Physics (Thomson-Brooks/Cole, Belmont, 2005), 3 ed.

[9] D. Hoekzema, E. van den Berg, G. Schooten y L. van Dijk, Physics education 42, 391 (2007).

[10] T. Mart, Phys. Educ. 56, 025012 (2021).

[11] K. Krijtenburg-Lewerissa, H.J. Pol, A. Brinkman y W.R. Van Joolingen, Physical review physics education research 13, 010109 (2017).

[12] H.K.E. Stadermann, E. van den Berg y M.J. Goedhart, Physical Review Physics Education Research 15, 010130 (2019).

[13] B. Winkler, P. Bitzenbaur y J-P. Meyn, Phys. Educ. 56, 065031 (2021).

[14] R.P. Feynman, R.B. Leighton y M.L. Sands, The Feynman lectures on physics (Addison-Wesley, Boston, 1963).

[15] T.S. Khun, The structure of scientific revolutions. (University of Chicago Press, Chicago, 1962).

[16] J. Quaglio, Revista Brasileira de Ensino de Fisica 43, e20210208 (2021). 
[17] M.A. Moreira, Boletín de estudios e investigación, disponible en: https://www.redalyc.org/articulo.oa?id=77 100606, accedido en set. 2021.

[18] L.C. McDermott, American Journal of Physics 69, 1127 (2001).

[19] L. Viennot, Raisonner en physique (De Boeck Supérieur, Paris, 1996).

[20] R. Gelman y R. Baillargeon, en: Handbook of child psychology: vol. 3, editado por P. Mussen, J.H. Flavell y E.M. Markman, Cognitive development (Wiley, New York, 1983), 4 ed., p. 167.

[21] D.D. Ebbing y S.D. Gammon, General Chemistry (Hughton Miffin, Boston, 2009). 11 ed.

[22] E. H. Wichmann, Quantum Physics (Mcgraw-Hill Co., New York, 1967).

[23] K. Krijtenburg-Lewerissa, H.J. Pol, A. Brinkman y W.R van Joolingen, Phys. Educ. 57, 025012 (2022). 